\title{
Shear bands in dense fault gouge
}

\author{
Nathalie Casas $^{1,2}{ }^{*}$, Guilhem Mollon ${ }^{1}$, and Ali Daouadji ${ }^{2}$ \\ ${ }^{1}$ Univ Lyon, INSA-Lyon, CNRS UMR5259, LaMCoS, 69621, France. \\ ${ }^{2}$ Univ. Lyon, INSA-Lyon, GEOMAS, EA7495, 69621, France.
}

\begin{abstract}
Earthquakes happen with frictional sliding, by releasing all the stresses accumulated in the prestressed surrounding medium. The geological fault gouge, coming from the wear of previous slips, acts on friction stability and plays a key role in this sudden energy release. A large part of slip mechanisms are influenced, if not controlled, by the characteristics and environment of this tribological "third body". A 2D granular fault (mm scale) is implemented with Discrete Element Modelling (DEM). A displacement-driven model with dry contact is studied to observe kinematics and properties of the slipping zone. Increasing the length of the granular media increases the slip needed to weaken the friction from friction peak to steadystate. Low-angle Riedel shear bands are mostly observed. Their number increases with the inter-particle friction coefficient, which also influences shear bands formation in their orientation angle (higher friction leads to higher angle with the main slip direction).
\end{abstract}

\section{Introduction}

During earthquakes, frictional sliding releases all stresses accumulated in the pre-stressed surrounding medium. Analogies with the concept of tribological triplet [1] are straightforward: between the two first bodies (i.e. rock walls constituting the fracture), the geological third body is composed by the granular material coming from the wear of previous slips. Characteristics of this third body play an important role in slip mechanisms. Studying the physics of slip triggering requires to account for granular behaviours within the shear fracture. Discrete Element Modelling (DEM) was first proposed by Cundall [2] in 1979, and has been applied several times in this context due to its ability to represent a wide range of materials of different scales inside granular faults [3, 4]. In this study, the DEM code MELODY 2D [5] is used to simulate a mature fault with cemented material between rock particles. To simulate this cement, we first realized a study with angular and faceted grains with cohesion law between particles [6]. Another approach is used here to model this cement with a matrix created by an assembly of small polygonal cells. This work is seen as the first step towards simulations considering a mixture between angular grains surrounded by polygonal cells representing a matrix of fines. This paper only focuses on this matrix and aims to present the shearing and slip behaviour of a granular medium composed only by conforming polygonal cells.

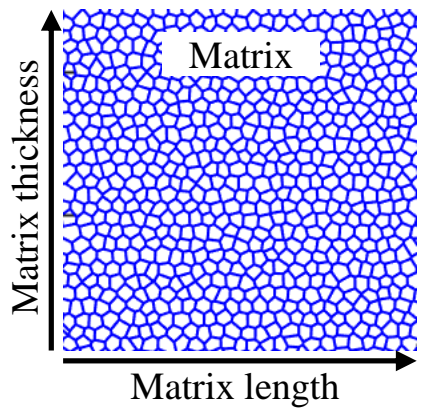

Fig. 1. Matrix shape and size, equivalent diameter $20 \mu \mathrm{m}$. Zoom in on a $500 \mu \mathrm{m} \times 500 \mu \mathrm{m}$ square part.

\section{Model description}

\subsection{Numerical geophysical third body}

A granular matrix is created with polygonal cells to represent a highly dense cement of particles. As the shape is polygonal, a Voronoï tessellation algorithm is used to create the cells with the appropriate size and shape. To create the matrix sample, a domain is considered, in which the size of cells and their perturbation (i.e. the homogeneity of the polygonal cells) are specified. The same size of cells is considered for each numerical experiment $\left(\emptyset_{e q}=20 \mu \mathrm{m}\right)$ and with the same perturbation of 0.5 , meaning that cells are not perfectly regular hexagons (Fig.1). The initial thickness of the layer remains constant in each simulation $(2 \mathrm{~mm})$ but different lengths of matrix are created in order to evaluate the influence of this parameter on shear localization and granular behaviour. The length is varied from 2 to $40 \mathrm{~mm}$.

\footnotetext{
* Corresponding author: nathalie.casas@insa-lyon.fr

A video is available at https://doi.org/10.48448/nm1s-hw69
} 


\subsection{Direct shear experiment with DEM}

The matrix is then introduced between two rock walls in order to realize direct shear experiments (Fig.2). The lower rock wall is fixed and normal stress (40 MPa) and slip velocity $(1 \mathrm{~m} / \mathrm{s})$ are imposed on the upper rock wall. Periodic boundary conditions are present on both rightand left-hand sides of the sample to maintain the continuity of the movement at large slips, but the upper rock wall can freely move in the y-direction to allow dilatancy. As geological third body is considered, the volumetric mass is chosen to correspond to Granite (i.e. $\left.2600 \mathrm{~kg} / \mathrm{m}^{3}\right)$. An interesting point is that the initial porosity within the gouge (i.e. percentage of voids) is negligible, with less than $1 \%$. This initial state of compaction is thus very close to intact rock.

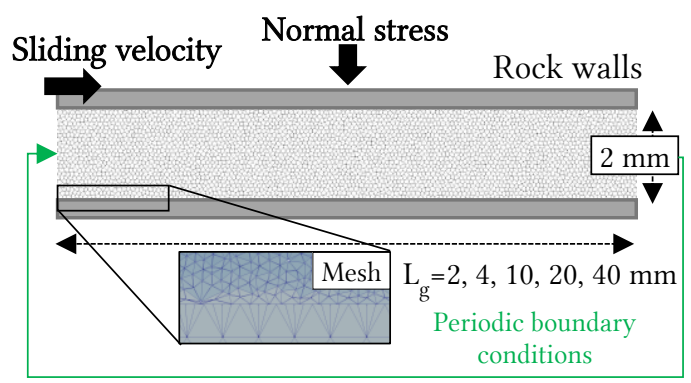

Fig. 2. DEM model of a direct shear of a granular matrix with MELODY 2D. Five different lengths of matrix created $(2,4,10$, $20 \& 40 \mathrm{~mm})$ with respectively $(11596,23174,58255,115825$ $\& 231671$ polygonal cells).

A dry contact model with rigid bodies is considered with no gravity (i.e. negligible in comparison to normal and deviatoric stresses). A Coulomb friction contact law is settled meaning that every contact depends on an interparticle friction $\mu_{i}$, numerical stiffnesses $k_{n}$ and $k_{t}$ (constant and equal to $1 \mathrm{e} 15 \mathrm{~N} / \mathrm{m}^{3}$ ) and a numerical damping $\gamma$ (numerically chosen and equal to 0.2 ). To avoid wall-slip effects with smooth boundaries, a certain cohesion is introduced at the interface between matrix and rock walls $(500 \mathrm{MPa})$. It enables to create a certain roughness by cementing particles to the top and bottom layer.

\section{Results \& discussion}

\subsection{Influence of model length}

In order to observe the influence of the length of the granular third body, five samples are generated: $\mathrm{L}_{2}(2 \mathrm{x} 2$ $\left.\mathrm{mm}^{2}\right), \mathrm{L}_{4}\left(4 \times 2 \mathrm{~mm}^{2}\right), \mathrm{L}_{10}\left(10 \times 2 \mathrm{~mm}^{2}\right), \mathrm{L}_{20}\left(20 \times 2 \mathrm{~mm}^{2}\right), \&$ $\mathrm{L}_{40}\left(40 \times 2 \mathrm{~mm}^{2}\right)$ with an inter-particle friction $\mu_{i}$ equal to 0.3 between cells.

As can be seen in (Fig.3), from 0 to point A, an elastic linear part is observed with the same behaviour for all samples. At point B (friction peak), almost all samples behave in the same way except for the $\mathrm{L}_{2}$ sample, which has a specific behaviour due to his particularly small size. Point $\mathrm{C}$ is very interesting, this is the decreasing friction point located at middle distance between friction peak and steady-state friction. An evolution of suddenness of friction weakening is observed (i.e. slip distance needed for friction to drop from $\mu_{p}$ to $\mu_{s s}$ ) and this area is also the place of maximum dilatancy. Increasing the length of the matrix softens the transition between $\mu_{p}$ to $\mu_{s s}$ until a certain limit. It also expands the dilation of the sample (Fig 3 (b)). Friction transition evolution stops evolving from after $\mathrm{L}_{20}$ (i.e. Representative Surface Element obtained for this gouge thickness, see the path of $\mathrm{L}_{40}$ in the Fig. 3 (a)). The same behaviour is obtained for dilation, increasing until $\mathrm{L}_{20}$. However, changing the gouge thickness or size of particles modifies the global stiffness of the granular media and thus could influence the RSE.

(a) Friction

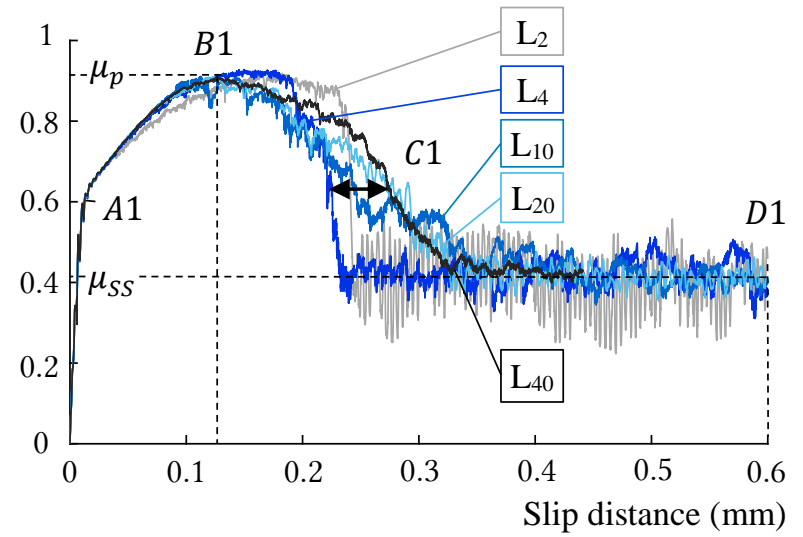

(b) Dilation

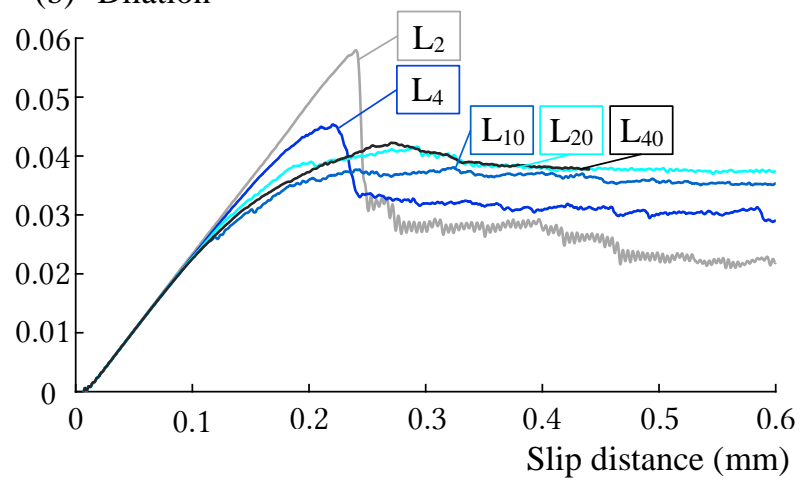

Fig. 3. (a) Friction coefficient as a function of the slip distance $(\mathrm{mm})$ for different sizes of models, $\mathrm{L}_{2}\left(2 \times 2 \mathrm{~mm}^{2}\right), \mathrm{L}_{4}\left(4 \times 2 \mathrm{~mm}^{2}\right)$, $\mathrm{L}_{10}\left(10 \times 2 \mathrm{~mm}^{2}\right), \mathrm{L}_{20}\left(20 \times 2 \mathrm{~mm}^{2}\right), \mathrm{L}_{40}\left(40 \times 2 \mathrm{~mm}^{2}\right) . \mu_{p}$ is the peak friction and $\mu_{s s}$ the steady state friction; (b) Dilation as a function of the slip distance $(\mathrm{mm})$ for the same sizes of models.

Fig. 4 presents the evolution of field of solid fraction for the $\mathrm{L}_{10}$ sample (ratio occupied by cells surface to the apparent surface of the granular layer). Until B1 in Fig. 4, several typical patterns of shear bands, called Riedel shear bands [7] appear. This pattern follows closely what is commonly observed in natural faults [8]. Between $\mu_{p}$ and $\mu_{s s}$, crack opening takes place in a preferred Riedel band $\mathrm{R}_{1}$ at $\mathrm{C} 1$, where a maximum dilatancy is observed (i.e. minimum solid fraction inside the Riedel shear). The augmentation of the length of the matrix does not seem to change the formation of observed Riedel shear bands. However, this increase creates a larger observation window displaying more Riedel bands within the sample. These bands are always inclined with the same orientation of $\approx 10^{\circ}$ from the main shear direction. They grow from the top surface to the bottom rock wall in the opposite direction of shearing. Steady state is reached with the 
formation of a localized horizontal shear band $\mathrm{S}$ at the top or bottom part of the granular media (D1 in Fig. 4).

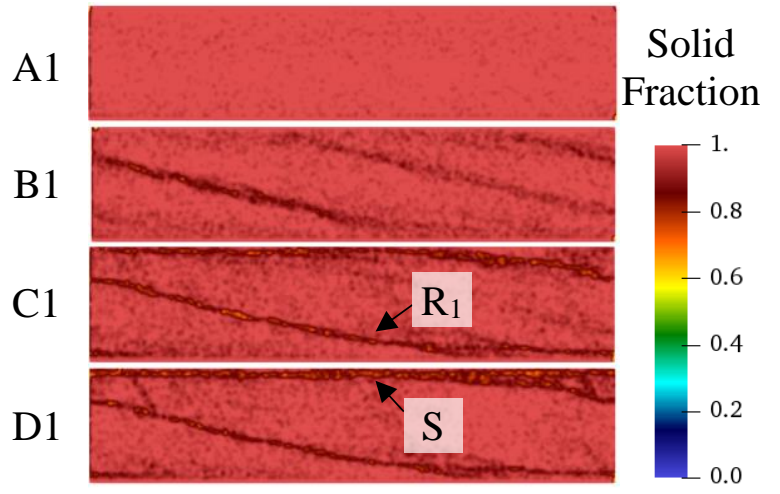

Fig. 4. Solid fraction as a function of a slip distance (mm) letter from figure $3, \mathrm{~L}_{10}$.

\subsection{Influence of inter-particle friction}

Numerical experiments are difficult to calibrate, as input data injected in the model are poorly documented in real granular media (i.e. inter-particle friction). A change in inter-particle friction coefficient from 0.3 to 0.6 can yield significant differences in friction peak values (Fig. 5 (a)) ( 0.9 vs 1.3 for $\mathrm{L}_{10}$ ). But both numerical experiments tend to the same averaged friction at steady state $\left(\mu_{s s}=0.42\right)$. A modification in inter-particle friction also changes the main orientation of Riedel shear bands $\left(\approx 16^{\circ}\right.$ from the horizontal shear direction with $\mu_{i}=0.6$ ).

(a) Friction

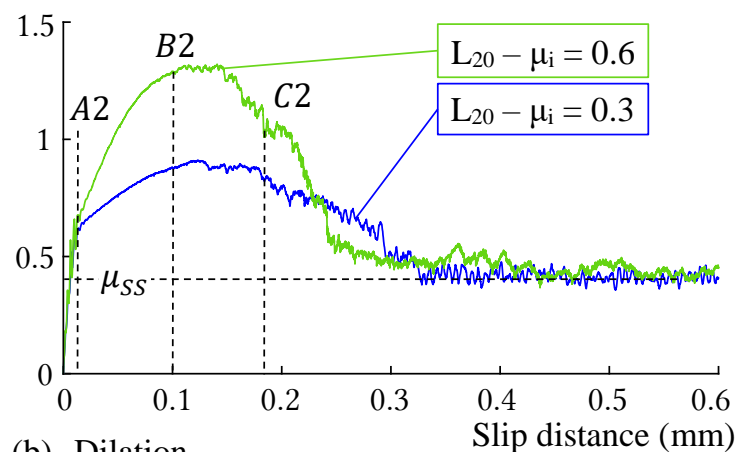

(b) Dilation

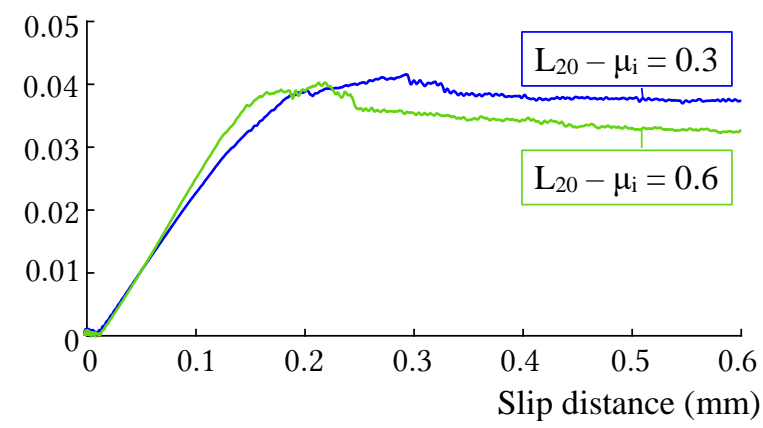

Fig. 5. (a) Friction coefficient as a function of the slip distance (mm) for different inter-particle friction $\left(\mu_{i}=0.3 \& 0.6\right)$, for L20 (the same friction peak difference is observed for the other lengths of models). (b) Dilation as a function of the slip distance $(\mathrm{mm})$ for the same cases.
Increasing inter-particle friction also seems to increase the number of Riedel bands formed at the beginning of friction peak (B2 in Fig. 5 \& Fig. 6) and within the decreasing friction part ( $\mathrm{C} 2$ in Fig. 6). This increase results in reducing the distance average $d$ between Riedel bands (Fig. 6). Higher angle Riedel bands $\left(\approx 45^{\circ}\right.$ from the horizontal shear direction) are also more pronounced with higher friction, (at C2 in Fig. 7).

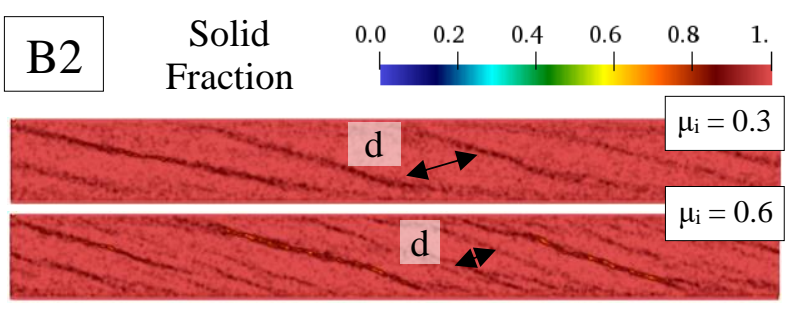

Fig. 6. Solid fraction at slip distance B2 for two different interparticle frictions $\left(\mu_{i}=0.3 \& 0.6\right), L_{20}$, letter from Fig. 5 .

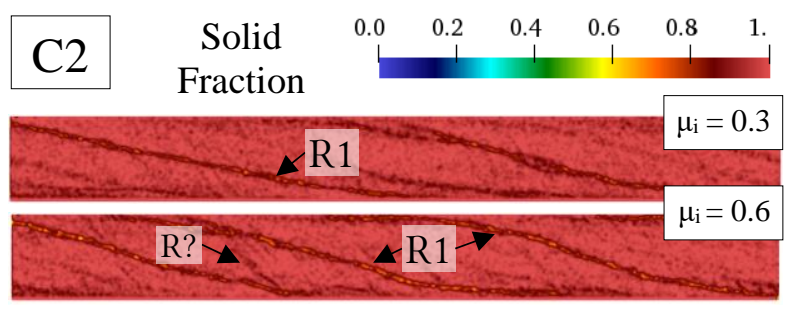

Fig. 7. Solid fraction at slip distance $\mathrm{C} 2$ for two different interparticle frictions $\left(\mu_{i}=0.3 \& 0.6\right), \mathrm{L}_{20}$, letter from Fig. 5 .

The two Riedel bands described as low-angle (R1) and high angle (R2) do not have the same growing pattern. They both depend on porosity-weakening and strain-rateweakening mechanisms [9]. Initial state of porosity is not modified here, but inter-particle friction plays a role on the rheology of the granular media, as it changes the contact between every polygonal cell. It may explain why high-angle Riedel bands are more pronounced with higher friction. The increasing number of Riedel bands with inter-particle friction can be explained with contact mechanics [10]. For the peak phase, when $\mu_{i}$ is low, slip may occur between polygonal cells, and particles are mobilized within the shear zone, with minimal dilation. With a higher $\mu_{i}$, sliding is partly inhibited and particles tend to separate leading to higher dilations, and thus in our case, higher number of Riedel bands (i.e. dilation in specific direction). However, at the end of friction peak, Riedel bands reduce into a more stable behaviour and total dilation tends to be higher for the case with lower friction.

As shown in Fig. 5, an interparticle friction coefficient of 0.6 also leads to a more sudden post-peak weakening, which is prone to switch the fault behaviour from a ductile aseismic response to a brittle seismic slip, depending on the stiffness of the surrounding medium.

\section{Conclusion}

Changing the length of the fault gouge sample does not inhibit the formation of shear bands but it modifies the rate of friction weakening with the slip displacement. An increase of inter-particle friction seems to influence shear bands formation with two main consequences. The 
orientation angle of Riedel bands from the horizontal slip displacement increases with the inter-particle friction (typical results from Mohr-Coulomb theory). Then, lowangle Riedel bands are mostly observed and their number increases with friction.

On-going studies are dedicated to mixing polygonal cells with angular grains in order to represent granitic particles with a cement (i.e. polygonal cells) between them. Different results are expected in terms of Riedel patterns as intern structure will be less homogeneous. A modification of matrix thickness could also influence the rheology of the fault.

\section{References}

[1] Y. Berthier, Experimental evidence for friction and wear modelling. Wear 139, 77 (1990)

[2] P.A. Cundall, O.D.L. Strack, A discrete numerical model for granular assemblies, Geotech. 29, 47 (1979)

[3] J.K. Morgan, M.S. Boettcher, Numerical simulations of granular shear zones using the distinct element method: 1. Shear zone kinematics and the micromechanics of localization, J. Geophys. Res. Solid Earth 104, 2703 (1999)

[4] B. Ferdowsi, M. Griffa, R.A. Guyer, P.A. Johnson, C. Marone, J. Carmeliet, Three-dimensional discrete element modeling of triggered slip in sheared granular media, Phys. Rev. E 89, 1 (2014)

[5] G. Mollon, A multibody meshfree strategy for the simulation of highly deformable granular materials, Int. J. Numer. Methods Eng. 108, 1477 (2016)

[6] N. Casas, G. Mollon, A. Daouadji, Cohesion and Initial Porosity of Granular Fault Gouges control the Breakdown Energy and the Friction Law at the Onset of Sliding, ESSOAR (in prep)

[7] J.S. Tchalenko, Similarities between shear zones of different magnitudes, Geol. Soc. Am. Bull. 81, 1625 (1970)

[8] Y. Katz, R. Weinberger, A. Aydin, Geometry and kinematic evolution of Riedel shear structures, Capitol Reef National Park, Utah, J. Struct. Geol. 26, 491 (2004)

[9] R.F. Katz, M. Spiegelman, B. Holtzman, The dynamics of melt and shear localization in partially molten aggregates, Nature 442, 2 (2006)

[10] J.K. Morgan, Numerical simulations of granular shear zones using the distinct element method: 2. Effects of particle size distribution and interparticle friction on mechanical behavior, J. Geophys. Res. Solid Earth 104, 2721 (1999) 